Paul Portner, Claudia Maienborn and Klaus von Heusinger (Eds.)

Semantics - Sentence and Information Structure 
This volume is part of a larger set of handbooks to Semantics

1 Semantics: Foundations, History and Methods

Klaus von Heusinger, Claudia Maienborn, Paul Portner (eds.)

2 Semantics: Lexical Structures and Adjectives

Claudia Maienborn, Klaus von Heusinger, Paul Portner (eds.)

3 Semantics: Theories

Claudia Maienborn, Klaus von Heusinger, Paul Portner (eds.)

$4 \quad$ Semantics: Noun Phrases and Verb Phrases

Paul Portner, Klaus von Heusinger, Claudia Maienborn (eds.)

5 Semantics: Sentence and Information Structure

Paul Portner, Claudia Maienborn, Klaus von Heusinger (eds.)

6 Semantics: Interfaces

Claudia Maienborn, Klaus von Heusinger, Paul Portner (eds.)

7 Semantics: Typology, Diachrony and Processing

Klaus von Heusinger, Claudia Maienborn, Paul Portner (eds.) 


\section{Semantics \\ Sentence and \\ Information Structure}

Edited by

Paul Portner

Claudia Maienborn

Klaus von Heusinger

DE GRUYTER

MOUTON 
ISBN 978-3-11-058722-7

e-ISBN (PDF) 978-3-11-058986-3

e-ISBN (EPUB) 978-3-11-058731-9

\section{Library of Congress Cataloging-in-Publication Data}

Names: Portner, Paul, editor. | Maienborn, Claudia, editor. | Heusinger, Klaus von, editor.

Title: Semantics : foundations, history and methods / edited by Paul Portner, Claudia Maienborn, Klaus von Heusinger.

Description: Berlin ; Boston : De Gruyter, [2019] | Series: Mouton reader | Includes bibliographical references and index.

Identifiers: LCCN 2018031290 (print) | LCCN 2018045977 (ebook) | ISBN

9783110589863 (electronic Portable Document Format (pdf) | ISBN

9783110587227 (print : alk. paper) | ISBN 9783110589863 (e-book pdf)| ISBN 9783110587319 (e-book epub)

Subjects: LCSH: Semantics.

Classification: LCC P325 (ebook) | LCC P325 .S37997 2019 (print) | DDC 401/.43--dc23

LC record available at https://lccn.loc.gov/2018031290

\section{Bibliographic information published by the Deutsche Nationalbibliothek}

The Deutsche Nationalbibliothek lists this publication in the Deutsche Nationalbibliografie; detailed bibliographic data are available in the Internet at http://dnb.dnb.de.

(C) 2019 Walter de Gruyter GmbH, Berlin/Boston

Cover image: anyaivanova / Getty Images Plus Typesetting: Integra Software Services Pvt. Ltd. Printing and binding: CPI books $\mathrm{GmbH}$, Leck

www.degruyter.com 